\section{Coupled rolling circle amplification loop- mediated amplification for rapid detection of short DNA sequences}

\author{
Jennifer Y. Marciniak ${ }^{1}$, Andrew C. Kummel2, Sadik C. Esener ${ }^{3}$, \\ Michael J. Heller1,3, and Bradley T. Messmer ${ }^{4}$ \\ ${ }^{1}$ Department of Bioengineering, ${ }^{2}$ Department of Chemistry and Biochemistry, \\ ${ }^{3}$ Department of Nanoengineering, and ${ }^{4}$ Rebecca and John Moores Cancer Center, \\ University of California San Diego, La Jolla, CA, USA
}

BioTechniques 45:275-280 (September 2008)

doi 10.2144/000112910

Circularizable oligonucleotide probes can detect short DNA sequences with single-base resolution at the site of ligation and can be amplified by rolling circle amplification (RCA) using strand displacing polymerases. A secondary amplification scheme was developed that uses the loop-mediated amplification reaction concurrent with RCA to achieve rapid signal development from the starting circular molecules. This isothermal reaction was found to be significantly faster than the comparable hyperbranching amplification method and could detect 100 circular copies in less than $1 \mathrm{~h}$.

\section{INTRODUCTION}

Rolling circle amplification (RCA) of circularizable oligonucleotides ("padlock" probes) is a sensitive method for the detection and amplification of short DNA sequences. The padlock probe (1) consists of a singlestranded oligonuceotide whose $5^{\prime}$ and $3^{\prime}$ ends hybridize to a target of interest and are then ligated to create a singlestranded circular DNA molecule, which is then a substrate for RCA $(2,3)$. The sensitivity of DNA ligase to mismatches and the fact that the ligated probe will remain hybridized to the target when subjected to stringent washing conditions result in a very high signal-to-noise ratio. As such, padlock probes are useful tools for the detection of gene sequences and variants because the target can be detected directly (2). Since the regions of the probe not involved in target binding are arbitrary, they can be designed to encode hybridization targets for differentiable probes or other sequence moieties that facilitate simultaneous multiplexed analysis $(4,5)$. Such strategies are generally less susceptible to cross-reactions than PCR (6).

Padlock probes and RCA have also been successfully coupled with fluores- for strand displacement, which bump the product produced by an inner primer, and the inner primers contain sequences of the sense and antisense strands of the target DNA that can fold over and be used for self-priming in later stages. The result is a family of concatemeric products. LAMP has been used to rapidly detect pathogens such as Salmonella and West Nile virus (20-24), and an accelerated form of LAMP, using additional loop primers, more than doubles the speed of the reaction (25). The isothermal nature of LAMP, similar to RCA, is very attractive for field applications in which thermal cycling methods such as PCR may be difficult to implement.

This paper describes a method that combines the benefits of the padlock probe and RCA with the speed of LAMP. First, a padlock probe is used to hybridize to the region of interest. After the padlock probe is ligated, the first of two loop primers, designed like the two inner primers of LAMP, primes $\mathrm{RCA}$. The RCA concatemer product is then multiply primed by the other loop primer, creating displacement products that undergo LAMP. The RCA-LAMP reaction is a very rapid amplification as compared with a hyperbranching reaction.

rapid solution phase assays, secondary amplifications schemes including circle-to-circle (9) and hyperbranching (4) [also known as cascade RCA (10) or ramification amplification (11-13)] have been developed. Circleto-circle amplification is a multiplicative process that requires several repetitions of a process that includes changes in temperature and addition of reagents. Hyperbranching uses two primers to initiate RCA and then prime a cascading series of displacement syntheses, as is done with wholegenome amplification (14-17), forming a branched DNA structure. It has been shown that ramification amplification can detect 10 target molecules in 90 $\min (11,18)$.

Quasi-exponential isothermal amplification using strand-displacing DNA polymerase has been achieved by loop-mediated isothermal amplification (LAMP) (19). The LAMP method uses four primers to specifically amplify a short region ( $100 \mathrm{bp})$ of DNA. The outer primers are used

\section{MATERIALS AND METHODS}

\section{Oligonucleotides}

All primers and padlock probes were purchased from Integrated DNA Technologies (Coralville, IA, USA). The sequences of all oligonucleotides used in this study are shown in Table 1. The padlock probe is diagrammed in Figure 1. The primers for hyperbranching are abridged versions of the LAMP primers.

\section{RCA-LAMP and Hyperbranching Procedure}

Hybridization and ligation of the padlock probe was carried out in T4 DNA ligase reaction buffer [50 $\mathrm{mM}$ Tris- $\mathrm{HCl}, 10 \mathrm{mM} \mathrm{MgCl} 2,1 \mathrm{mM}$ ATP, $10 \mathrm{mM}$ dithiothreitol, $25 \mu \mathrm{g} / \mathrm{mL}$ bovine serum albumin (BSA); New 


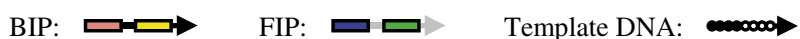

Padlock Probe:

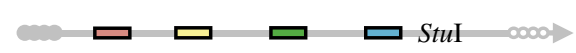

1. The padlock probe hybridizes to DNA sequence of interest, and its $5^{\prime}$ and $3^{\prime}$ ends are ligated together. If the padlock probe is not ligated, the primers cannot begin replication of any long strands of DNA.
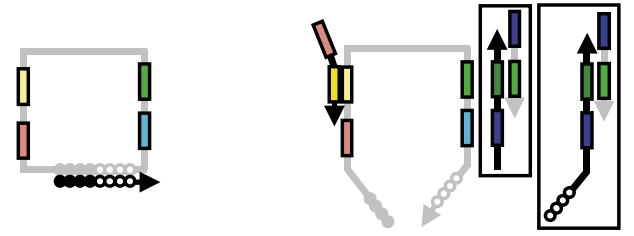

2. BIP binds to the ligated padlock probe and begins RCA.

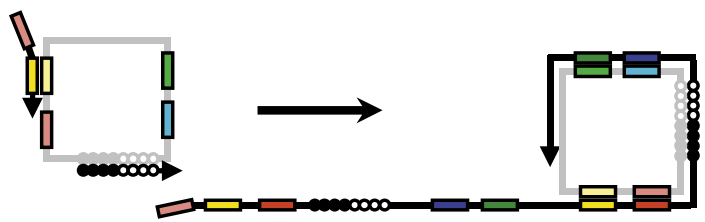

3. FIP will then bind to RCA product and start replication.

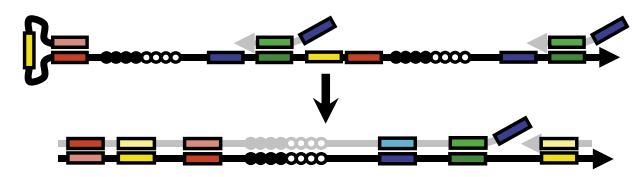

4. The product that extends from FIP will be displaced by the product from the FIP "behind" it, and the single-stranded DNA will hybridize to parts of itself. The $3^{\prime}$ end will begin replication.

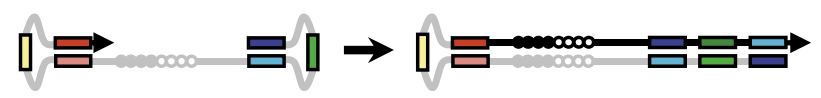

5. BIP can bind to the loop in the resulting structure and begin replication while displacing the strand that is already hybridized.

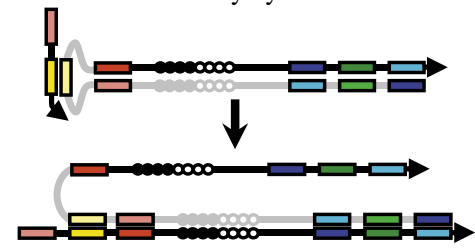

6. The $3^{\prime}$ end of the single-stranded DNA will fold over, hybridize to itself, and begin replication, displacing the strand that was just synthesized.

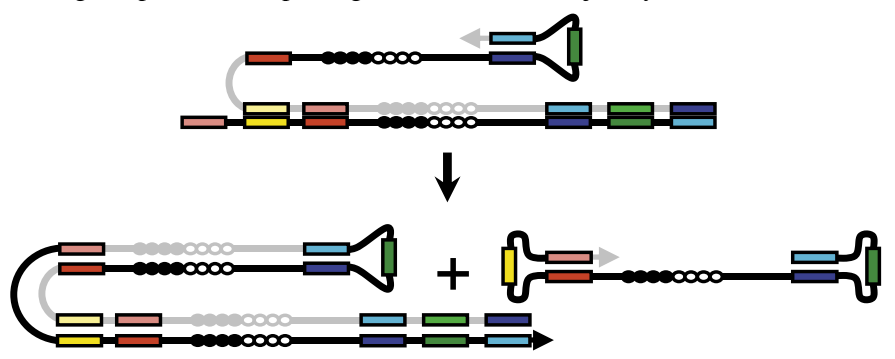

7. Now, FIP can bind to the single-stranded loop, and the process continues.

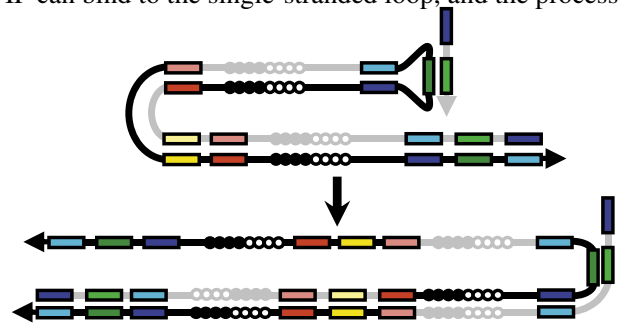

8. The $3^{\prime}$ end of the single-stranded DNA strand will fold over and begin replication. The process continues as long as reagents are available.

Several strands will slowly get longer and longer while shorter strands keep getting displaced and become longer strands.

Figure 1. Representation of rolling circle amplification loop-mediated isothermal amplification (RCA-LAMP). The $3^{\prime}$ ends are marked by an arrow. The padlock probe hybridizes to the sequence of interest and is ligated. RCA will only proceed from the BIP primer if the padlock probe has been ligated, otherwise the probe will be made double-stranded, and the reaction terminates. The FIP primer binds each concatemer within the RCA product, initiates DNA polymerization, and displaces the newly synthesized strand upstream. The displacement products then undergo the LAMP process. The LAMP product is encoded such that the sequence amplified is continuous only after padlock probe ligation (diagrammed in Step no. 1).

Table 1. Sequence of Padlock Probes, DNA Sequence of Interest (Template), and Primers

\section{Oligonucleotides Sequences}

F (hyperbranching primer) 5'-GCATTATCGATCAGTACCAGT-3'

$B$ (hyperbranching primer) 5'-ACGGTGACAATAGAGAAGAC-3'

FIP (RCA-LAMP primer)

5'-GTGGCGATAATTTCACCGGCTTTTGCATTATCGATCAGTACCAGT-3'

BIP (RCA-LAMP primer)

5'-GTTCGGGCAATTCGTTATTGGCTTTTACGGTGACAATAGAGAAGAC-3'

Template for $\mathrm{p} 53$

5'-GGGCGGCATGAACCGGAGGCCCATCCTCACC-3'

Padlock probe for $\mathrm{p} 53$

\section{5'-GGTTCATGCCGCCCGTTCGGGCAATTCGTTATTGGCCCCTATAGTGAGTCGTATTAGTCTTCTCTATTGT-} CACCGTACATCTCGGAATCAAGCTGGCATTATCGATCAGTACCAGTGTAGTACAGCAGCAGCATTGCCGGTGAAATTATCGCCACAGGCCTTTAAATATTCTCGAGGGTGAGGATGGGCCTCC-3'

BIP binds to the sequence that is underlined in bold. FIP binds to the complement of the sequence that is underlined. The Stul recognition sequence is denoted in italics, and the portion of the padlock probe that binds to the sequence of interest or template is in bold. RCA-LAMP, rolling circle amplification loop-mediated isothermal amplification. 


\begin{tabular}{|c|c|c|c|c|c|}
\hline FIP & & + & & + & + \\
\hline BIP & + & 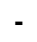 & + & + & + \\
\hline Padlock Probe & - & + & + & + & + \\
\hline
\end{tabular}

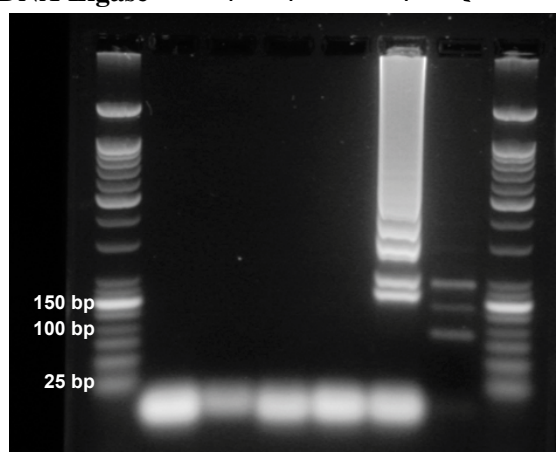

Figure 2. Rolling circle amplification loop-mediated isothermal amplification (RCA-LAMP) gel electrophoresis. All reactions were run for 30 min with $10^{6}$ DNA circles, where appropriate. Reaction products were run on a $2.2 \%$ agarose gel. The outside lanes are a $25 / 100 \mathrm{bp}$ mixed DNA ladder. Both primers are required for product formation, as is ligation of the padlock probe. The last sample shows a StuI restriction digest of the RCA-LAMP product, confirming that the high molecular weight products are the expected concatemer LAMP products.

England Biolabs, Ipswich, MA, USA] with T4 DNA ligase (New England Biolabs) for $2 \mathrm{~h}$ at $37^{\circ} \mathrm{C}$. The $25 \mu \mathrm{L}$ RCA-LAMP reaction mixture contains $1 \mathrm{M}$ betaine (Sigma Aldrich, St. Louis, MO, USA), $1 \times$ ThermoPol Buffer [20 $\mathrm{mM}$ Tris- $\mathrm{HCl}, 10 \mathrm{mM}\left(\mathrm{NH}_{4}\right)_{2} \mathrm{SO}_{4}, 10$ $\mathrm{mM} \mathrm{KCl}, 2 \mathrm{mM} \mathrm{MgSO}$, $0.1 \%$ Triton $\mathrm{X}-100$; New England Biolabs] supplemented with additional $\mathrm{MgSO}_{4}$ to bring the concentration to $10 \mathrm{mM}, 1.4$ $\mathrm{mM}$ dNTP (New England Biolabs), 40 pmol each primer, and $8 \mathrm{U}$ Bst polymerase (New England Biolabs). Mineral oil was added to each of the reactions to prevent contamination. Hyperbranching was performed with the same conditions but with the $\mathrm{F}$ and $\mathrm{B}$ primers. The reaction was carried out at $62^{\circ} \mathrm{C}$ in an Mx3005P Real-Time PCR system (Stratagene, La Jolla, CA, USA) with SYBR Green (Invitrogen, Carlsbad, CA, USA) as a measure of the reaction progression. Stratagene reference dye (ROX) was used to normalize well-to-well differences. The threshold time is defined as the time it takes for the baseline fluorescence to double. The final products of the RCA-LAMP reaction were visualized in a $2 \%$ agarose gel containing $1 \times$ GelRed (Biotium, Hayward, CA, USA) using a high performance transilluminator and DigiDoc-It from UVP (Upland, CA, USA).

\section{Isolation of Single-stranded Circles}

Pure circles were prepared by ligating the padlock probe, then eliminating excess DNA using Exonuclease III and Exonuclease $\mathrm{T}$ (both from New England Biolabs). The padlock probe $(100 \mathrm{nM})$ was hybridized to the template $(300 \mathrm{nM})$ and then ligated using T4 DNA ligase (New England Biolabs) in T4 ligase buffer for $2 \mathrm{~h}$ at $37^{\circ} \mathrm{C}$. The DNA was then concentrated using a Nucleotide Cleanup kit (Qiagen, Valencia, CA, USA) and eluted with water. Exonuclease III was used to cleave nucleotides from doublestranded DNA (i.e., the unligated padlock probe that had hybridized to the template DNA). The Exo III reaction was in NEBuffer $1(10 \mathrm{mM}$ Bis-Tris-propane- $\mathrm{HCl}, 10 \mathrm{mM} \mathrm{MgCl}$, $1 \mathrm{mM}$ dithiothreitol; New England Biolabs) and incubated at $37^{\circ} \mathrm{C}$ for 1 h. The enzyme was inactivated at $70^{\circ} \mathrm{C}$ for 20 min, and the Nucleotide Cleanup kit was used again to prepare the DNA for the next step.

Exonuclease $\mathrm{T}$ was then used to digest single-stranded oligonucleotides (i.e., the template and the unhybridized, unligated padlock probe). The reaction contained the DNA, ExoT, and NEBuffer $4(20 \mathrm{mM}$ Tris-acetate, $50 \mathrm{mM}$ potassium acetate, $10 \mathrm{mM}$ magnesium acetate, $1 \mathrm{mM}$ dithiothreitol; New England Biolabs) and was kept at $25^{\circ} \mathrm{C}$ for $1 \mathrm{~h}$ before heat inactivation of the enzyme at $65^{\circ} \mathrm{C}$ for $20 \mathrm{~min}$. After a final cleanup, the isolated circles were verified using a $10 \%$ Tris/Borate/EDTA (TBE)urea denaturing polyacrylamide gel (Invitrogen), and the DNA concentration was measured on the NanoDrop ND-1000 UV-Vis spectrophotometer

\section{Short Technical Reports}

(NanoDrop Technologies, Wilmington, DE, USA).

\section{RESULTS AND DISCUSSION}

The RCA-LAMP amplification scheme is illustrated in Figure 1. The padlock probe hybridizes to the sequence of interest, and the $5^{\prime}$ and $3^{\prime}$ ends are ligated to each other resulting in a continuous single-stranded DNA circle. The BIP primer hybridizes to the padlock probe and primes RCA. The FIP primer can bind to several spots on the resulting strand, begin replication at all of those spots, and displace the strands in front. The LAMP primers fold back upon the strand that they just synthesized, and when the $3^{\prime}$ end of a single-stranded piece of DNA folds back upon itself, it can begin replication of its own strand. Finally, the single-stranded loop that is formed when the 3' end folds back upon itself contains the sequence that either one or the other primer can bind to and continue replication and displacement of the DNA.

Figure 2 shows the result of the RCA-LAMP reaction with positive and negative controls. The reactions were run for $30 \mathrm{~min}$. The lane containing the reaction without the FIP primer likely resulted in RCA, but because the reaction was only run for $30 \mathrm{~min}$, there was not enough product to visualize on the gel. The results show that the ligated padlock probe and both primers are necessary for the reaction to occur, and the reaction yields are substantial. As the displaced reaction products lengthen, several products in different states of amplification produce a smear. The inclusion of the StuI recognition sequence in the padlock probe allowed for an StuI digest to test for the correct 


\section{Short Technical Reports}
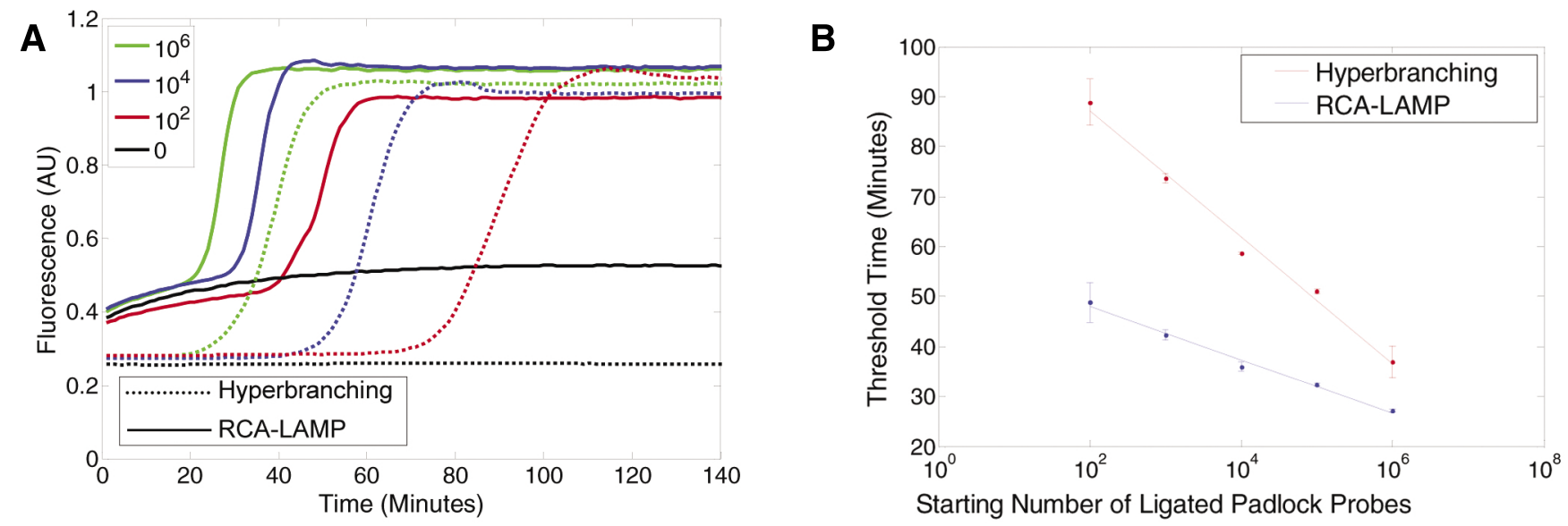

Figure 3. Comparison of rolling circle amplification loop-mediated isothermal amplification (RCA-LAMP) reaction to hyperbranching. (A) RCALAMP and hyperbranching reactions were performed in parallel on a real-time instrument with varying numbers of circular template molecules. (B) The threshold time [determined by the instrument's real-time PCR cycle threshold $\left(\mathrm{C}_{\mathrm{T}}\right)$ determination algorithms] as a function of template circles is shown. The relationship between time to threshold and the template copy number is exponential as anticipated. AU, arbitrary units.

amplification products. Unlike the $12 \mu \mathrm{L}$ that were loaded into the wells from each reaction, only $2 \mu \mathrm{L}$ of the RCA-LAMP reaction was used for the StuI digest to ensure more complete digestion of the larger fragments. The expected products of the digest in base pairs are 87,186 , and approximately 71 and 23 because of the single-stranded loops. The gel shows digestion products around the expected 87 and 186, and some larger fragments that suggest incomplete digestion.

Target detection using padlock probes amplified by hyperbranching has been demonstrated $(11-13,18)$.
RCA-LAMP was compared with the established hyperbranching process using the same padlock probe and hyperbranching primers that recognized the same sites as the LAMP primers. Figure 3 shows that the RCA-LAMP reaction and the hyperbranching reactions can amplify a given number

\section{Free Subscription}

BioTechniques is the first peer-reviewed journal with

open access to the whole of the life science community.

$100 \%$ life science

$100 \%$ peer-reviewed methods

$100 \%$ "everyday" practical information

Sign up for free at

www.BioTechniques.com/subscribe

\section{BioTechnioues}

Connecting. Informing. Advancing. For 25 Years.

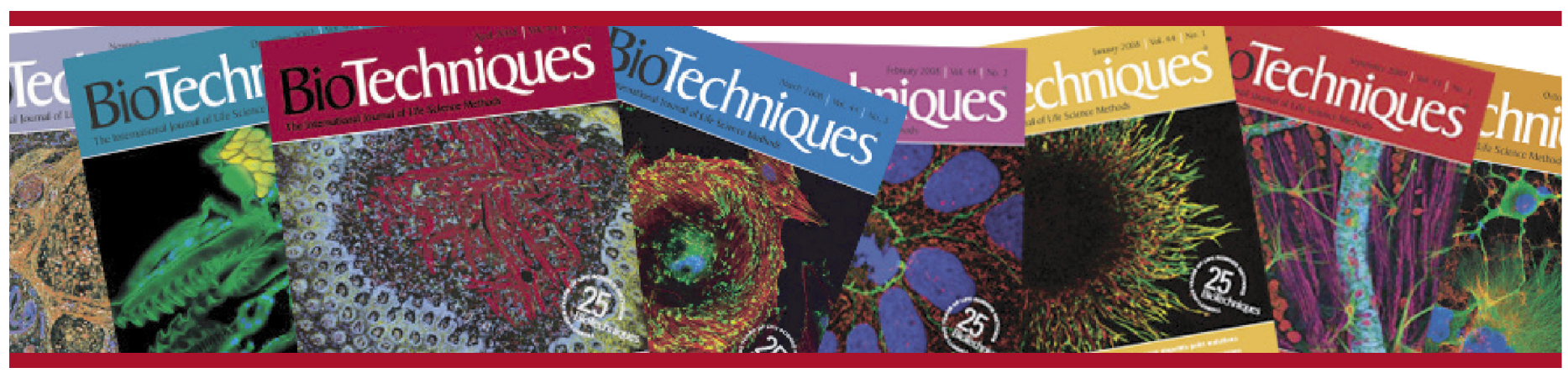




\section{Short Technical Reports}

of DNA circles in a reproducible manner. When the number of starting ligated circles is titrated (Figure 3A), each 10 -fold decrease in the number of starting circles shows a consistent delay in amplification time and a rapid amplification from a few number of DNA circles; namely 100. As the number of circles was titrated below 100 , the results became inconsistent (data not shown). The RCA-LAMP reaction consistently amplified ligated circles more rapidly than the hyperbranching reaction. When the reaction begins, there is a gradual increase in background fluorescence associated with the RCA-LAMP reaction, most clearly observed with the negative control. This is thought to be a product of the long primers folding and forming stem-loop structures that the polymerase can extend to the end of the $5^{\prime}$ end of each primer. This activity levels out after about $40 \mathrm{~min}$ and is clearly distinguishable from the positive controls.
The highly prolific RCA-LAMP amplification process is very sensitive to contamination, particularly since the early displacement products can seed the reaction. It is essential to ensure careful procedures to minimize contamination, as with PCR. For this study, careful separation of each step of the process (i.e., the amplification products were kept away from the reagents) and the addition of a mineral oil helped to control the spread of the product. Another technique that can be used is to add dUTPs instead of dTTPs to the reaction and treat the tube with uracil-DNA N-glycosylase to eliminate contaminants from previous reactions. Also, large amounts of unligated padlock probes can lead to inappropriate amplification as seen with the hyperbranching reaction (26-28). Depending on the application, this can be dealt with by first purifying the target and then running the amplification reaction $(12,13)$.
Nonspecific DNA sequences may also trigger the amplification reaction to occur. This is another reason to purify the ligated circles before running the RCA-LAMP reaction. In the cases where a nonspecific DNA sequence has triggered amplification, the StuI digest did not result in the proper restriction fragments. And since padlock probes can encode a variety of secondary detection moieties such as restriction sites or sequences for fluorescent probes to hybridize to, these false positives can be distinguished from true positive results.

The RCA-LAMP reaction couples the specificity of padlock probes with a very rapid isothermal amplification process that allows for rapid detection of a DNA sequence of interest from a relatively small number of molecules. Like hyperbranching, this method combines the specificity and designability of a padlock probe with a secondary amplification scheme,

\section{Connecting. Informing. Advancing. For 25 Years. BiOlechniQues}

\section{BioTechniques Weekly}

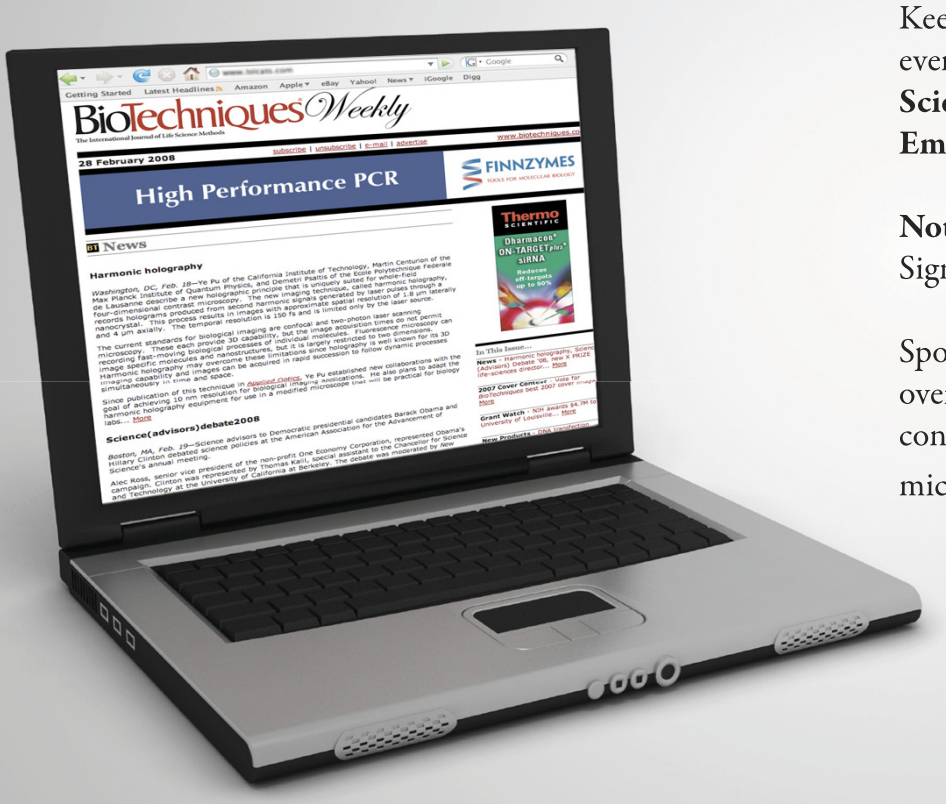

Keep up-to-date with this free weekly electronic newsletter delivered every Thursday to BioTechniques subscribers. Coverage includes:

Science news - Recent top grants - New products • Employment opportunities - Event calendar - and more...

Not a subscriber?

Sign up free at www.BioTechniques.com/newsletters

Sponsorship opportunities are available. Your sponsorship will reach over 75,000 subscribers per newsletter. To sponsor a newsletter, please contact Michelle Graves.

michelle.graves@informa.com |+44 (20) 70176785 
which may allow a single reaction containing one set of primers and multiple padlock probes to probe for several sequences of interest (29). Each padlock probe can be designed to probe for distinct sequences, and because the sequences of the padlock probes are different from each other, the products from each of these padlock probes can be distinguished by unique hybridization probes. By combining the ability of padlock probes to probe for short sequences of DNA with the very rapid LAMP reaction, the RCA-LAMP reaction may be particularly useful for frontline genetic detection applications, such as the detection of bioweapons or infectious agents.

\section{ACKNOWLEDGMENTS}

This work was supported by a National Institutes of Health (NIH) grant from the National Cancer Institute (NCI) Alliance for Nanotechnology in Cancer (no. U54 CA119335). We would like to thank Benjamin Sullivan and Jason Steiner for helpful discussions pertaining to this manuscript and Nima Farahbakhsh for the time he spent on this project. This paper is subject to the NIH Public Access Policy.

\section{COMPETING INTERESTS STATEMENT}

The authors declare no competing interests.

\section{REFERENCES}

1. Nilsson, M., H. Malmgren, M. Samiotaki, M. Kwiatkowski, B.P. Chowdhary, and U. Landegren. 1994. Padlock probes: circularizing oligonucleotides for localized DNA detection. Science 265:2085-2088.

2. Baner, J., M. Nilsson, M. Mendel-Hartvig, and U. Landegren. 1998. Signal amplification of padlock probes by rolling circle replication. Nucleic Acids Res. 26:5073-5078.

3. Fire, A. and S.Q. Xu. 1995. Rolling replication of short DNA circles. Proc. Natl. Acad. Sci. USA 92:4641-4645.

4. Lizardi, P.M., X. Huang, Z. Zhu, P. BrayWard, D.C. Thomas, and D.C. Ward. 1998. Mutation detection and single-molecule counting using isothermal rolling-circle amplification. Nat. Genet. 19:225-232.
5. Nilsson, M., K. Krejci, J. Koch, M. Kwiatkowski, P. Gustavsson, and U. Landegren. 1997. Padlock probes reveal single-nucleotide differences, parent of origin and in situ distribution of centromeric sequences in human chromosomes 13 and 21. Nat. Genet. 16:252-255.

6. Landegren, U. and M. Nilsson. 1997. Locked on target: strategies for future gene diagnostics. Ann. Med. 29:585-590.

7. Christian, A.T., M.S. Pattee, C.M. Attix, B.E. Reed, K.J. Sorensen, and J.D. Tucker. 2001. Detection of DNA point mutations and mRNA expression levels by rolling circle amplification in individual cells. Proc. Natl. Acad. Sci. USA 98:14238-14243.

8. Zhong, X.B., P.M. Lizardi, X.H. Huang, P.L. Bray-Ward, and D.C. Ward. 2001. Visualization of oligonucleotide probes and point mutations in interphase nuclei and DNA fibers using rolling circle DNA amplification. Proc. Natl. Acad. Sci. USA 98:3940-3945.

9. Dahl, F., J. Baner, M. Gullberg, M. MendelHartvig, U. Landegren, and M. Nilsson. 2004. Circle-to-circle amplification for precise and sensitive DNA analysis. Proc. Natl. Acad. Sci. USA 101:4548-4553.

10. Thomas, D.C., G.A. Nardone, and S.K. Randall. 1999. Amplification of padlock probes for DNA diagnostics by cascade rolling circle amplification or the polymerase chain reaction. Arch. Pathol. Lab. Med. 123:1170-1176.

11. Zhang, D., J. Wu, F. Ye, T. Feng, I. Lee, and B. Yin. 2006. Amplification of circularizable probes for the detection of target nucleic acids and proteins. Clin. Chim. Acta 363:61-70.

12. Zhang, D.Y., M. Brandwein, T. Hsuih, and H.B. Li. 2001. Ramification amplification: a novel isothermal DNA amplification method. Mol. Diagn. 6:141-150.

13. Zhang, D.Y., W. Zhang, X. Li, and Y. Konomi. 2001. Detection of rare DNA targets by isothermal ramification amplification. Gene 274:209-216.

14. Dean, F.B., S. Hosono, L. Fang, X. Wu, A.F. Faruqi, P. Bray-Ward, Z. Sun, Q. Zong, et al. 2002. Comprehensive human genome amplification using multiple displacement amplification. Proc. Natl. Acad. Sci. USA 99:5261-5266.

15. Dean, F.B., J.R. Nelson, T.L. Giesler, and R.S. Lasken. 2001. Rapid amplification of plasmid and phage DNA using Phi 29 DNA polymerase and multiply-primed rolling circle amplification. Genome Res. 11:1095-1099.

16. Hosono, S., A.F. Faruqi, F.B. Dean, Y. Du, Z. Sun, X. Wu, J. Du, S.F. Kingsmore, et al. 2003. Unbiased whole-genome amplification directly from clinical samples. Genome Res. 13:954-964.

17. Lasken, R.S. and M. Egholm. 2003. Whole genome amplification: abundant supplies of DNA from precious samples or clinical specimens. Trends Biotechnol. 21:531-535.

18. Yi, J., W. Zhang, and D.Y. Zhang. 2006. Molecular Zipper: a fluorescent probe for realtime isothermal DNA amplification. Nucleic Acids Res. 34:e81.

19. Notomi, T., H. Okayama, H. Masubuchi, T. Yonekawa, K. Watanabe, N. Amino, and T. Hase. 2000. Loop-mediated isothermal amplification of DNA. Nucleic Acids Res. 28:E63.
20. Mori, Y., K. Nagamine, N. Tomita, and T. Notomi. 2001. Detection of loop-mediated isothermal amplification reaction by turbidity derived from magnesium pyrophosphate formation. Biochem. Biophys. Res. Commun. 289:150-154

21. Nagamine, K., K. Watanabe, K. Ohtsuka, T. Hase, and T. Notomi. 2001. Loop-mediated isothermal amplification reaction using a nondenatured template. Clin. Chem. 47:1742-1743.

22. Parida, M., G. Posadas, S. Inoue, F. Hasebe, and K. Morita. 2004. Real-time reverse transcription loop-mediated isothermal amplification for rapid detection of West Nile virus. J. Clin. Microbiol. 42:257-263.

23. Hara-Kudo, Y., M. Yoshino, T. Kojima, and M. Ikedo. 2005. Loop-mediated isothermal amplification for the rapid detection of Salmonella. FEMS Microbiol. Lett. 253:155-161.

24. Ohtsuka, K., K. Yanagawa, K. Takatori, and Y. Hara-Kudo. 2005. Detection of Salmonella enterica in naturally contaminated liquid eggs by loop-mediated isothermal amplification, and characterization of Salmonella isolates. Appl. Environ. Microbiol. 71:6730-6735.

25. Nagamine, K., T. Hase, and T. Notomi. 2002. Accelerated reaction by loop-mediated isothermal amplification using loop primers. Mol. Cell. Probes 16:223-229.

26. Alsmadi, O.A., C.J. Bornarth, W. Song, M. Wisniewski, J. Du, J.P. Brockman, A.F. Faruqi, S. Hosono, et al. 2003. High accuracy genotyping directly from genomic DNA using a rolling circle amplification based assay. BMC Genomics 4:21.

27. Faruqi, A.F., S. Hosono, M.D. Driscoll, F.B. Dean, O. Alsmadi, R. Bandaru, G. Kumar, B. Grimwade, et al. 2001. High-throughput genotyping of single nucleotide polymorphisms with rolling circle amplification. BMC Genomics 2:4.

28. Pickering, J., A. Bamford, V. Godbole, J. Briggs, G. Scozzafava, P. Roe, C. Wheeler, F. Ghouze, and S. Cuss. 2002. Integration of DNA ligation and rolling circle amplification for the homogeneous, end-point detection of single nucleotide polymorphisms. Nucleic Acids Res. 30:e60.

29. Mori, Y., T. Hirano, and T. Notomi. 2006 Sequence specific visual detection of LAMP reactions by addition of cationic polymers. BMC Biotechnol. 6:3.

Received 26 March 2008; accepted 9 June 2008.

Address correspondence to Bradley Messmer, Rebecca and John Moores Cancer Center, 3855 Health Science Drive, University of California San Diego, La Jolla, CA 920930815,USA.e-mail:bmessmer@ucsd.edu

To purchase reprints of this article, contact: Reprints@BioTechniques.com 